\title{
Filler-filler interactions and viscoelastic behavior of polymer nanocomposites
}

\author{
E. Chabert ${ }^{\mathrm{a}, \mathrm{b}, *}$, M. Bornert ${ }^{\mathrm{b}}$, E. Bourgeat-Lami ${ }^{\mathrm{c}}$, J.-Y. Cavaillé $^{\mathrm{a}}$, \\ R. Dendievel ${ }^{\mathrm{d}}$, C. Gauthier ${ }^{\mathrm{a}}$, J.L. Putaux ${ }^{\mathrm{e}}$, A. Zaoui ${ }^{\mathrm{b}}$

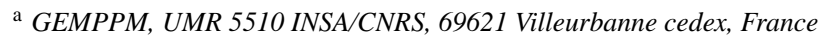 \\ b LMS, UMR 7649 École Polytechnique/CNRS, 91128 Palaiseau cedex, France \\ ' $L C P P$, UMR 140 CPE/CNRS, 69616 Villeurbanne cedex, France \\ d GPM2, UMR 5010 INPG/CNRS, BP 46, 38042 St Martin d'Hères cedex, France \\ e CERMAV, UPR 5301 CNRS, BP 53, 38041 Grenoble cedex 9, France
}

\begin{abstract}
This work presents the main results obtained within a project on mechanical properties of polymer based nanocomposites. The specific point was how to analyze and model the filler-filler interactions in the description of the viscoelastic behavior of these materials. This paper aims at presenting the general strategy used by the different partners to address this question, together with original experimental results and micro-mechanical modeling. Different nanocomposite materials were fabricated using the latex route, leading to random dispersions of rigid submicronic particles (PS = polystyrene, silica) in a flexible polybutylacrylate matrix at various volume fractions. In addition, encapsulated silica particles in a styrene-acrylate copolymer were produced, leading, after film formation, to a limited number of contacts between silica fillers. The processing route of these encapsulated particles was optimized and the resulting morphology was analyzed by TEM experiments. In the case of random mixtures, a strong effect of reinforcement appears in the rubbery field of the soft phase when the filler content is above a critical fraction (percolation threshold). The reinforcement in the rubbery plateau can be still exacerbated in the case of the PS particles if the material undergoes a heat treatment above the main relaxation of the PS phase. These experimental results illustrate the difference between geometrical percolation (when particles are just in contact) and mechanical percolation (with strong interactions between the fillers). The comparison of the results for PS and silica fillers shows once more that the strength of the interactions plays an important role. To account for the whole set of experimental data, two ways of modeling were explored: (i) homogenization methods based on generalized self-consistent schemes and (ii) a discrete model of spheres assembly which explicitly describes the ability of the contacts to transmit efforts.
\end{abstract}

Keywords: Nanocomposites; Mechanical properties; Percolation; Discrete modeling approach; Self-consistent modeling; Cryo TEM

\section{Introduction}

Among the several reasons to incorporate fillers into polymers (cost reduction, improvement of some physical properties such as flame retardancy or barrier properties) mechanical reinforcement is expected [1]. Traditional fillers display average characteristic sizes in the range of several microns. However, due to the development of nanosized fillers, the specific influence of the nanometric size in the reinforcement mechanisms has to be addressed. Composite materials

\footnotetext{
* Corresponding author. Tel: +33 169333318; fax:+33 169333026.

E-mail address: chabert@1ms.polytechnique.fr (E. Chabert).
}

based on nano-sized fillers, the so-called nanocomposites, are presently studied especially because they may have unusual combinations of properties [2-6]. These unusual properties may be a consequence of the extremely large specific interfacial area (hundreds of $\mathrm{m}^{2} / \mathrm{g}$ ). They may be also related to the very short distances between the reinforcing fillers (about $10^{-8} \mathrm{~m}$ ), that may become close to the characteristic size of the macromolecular coils. In addition, some years ago, we showed that drastic reinforcing effects may be observed at very low volume fractions for fillers with very large aspect ratio, when the percolation of the fillers occurs [7].

However, the processing of composite materials that only differ by the size of the fillers (keeping constant the dispersion state and surface properties) is not a simple task. In or- 
der to by-pass this difficulty, experimental data may be compared to theoretical predictions that are developed to account for the mechanical behavior of heterogeneous materials. Parameters which play a role for the mechanical properties of a filled polymer (elastic modulus for instance) are the following: (i) the (visco)elastic properties of its constitutive phases, (ii) the volume fraction of filler, (iii) the morphology (i.e., shape, aspect ratio, and distribution of the filler within the polymeric matrix) and (iv) the interactions between fillers and between filler and matrix. Various models have been proposed in the literature to understand the complex interplay between these parameters and to display a prediction of the elastic moduli of polymer composites. Moreover, it is generally accepted that these elastic calculations can be extended to the description of the viscoelasticity of filled polymers through the correspondence principle of Hashin [8]. In fact, the classical models, at least in their former developments, ignore direct interactions between fillers: fillers are considered as a homogeneous phase which interacts with the matrix or, at most, with the effective medium, and thus these models may be unefficient when the interactions between the fillers themselves rule the mechanical response of the composite. Moreover, they cannot simply account for the percolation of rigid fillers within a soft matrix.

The work presented in the following has been performed in the framework of a collaboration between several laboratories. The general strategy was first to elaborate model nanocomposites with controlled morphologies, to characterize their mechanical behavior in the linear domain and to develop mechanical models adapted to describe their behaviors. The first part of the paper presents the fabrication route to process polymers filled with spherical particles with diameters in the nanometer range. In order to understand reinforcement mechanisms depending on connectivity or on the aggregation state, we have focused on spherical fillers to avoid orientation effects. Materials were produced with different dispersion states: nanocomposites obtained from a mixture of hard/soft latex particles and nanocomposites made of encapsulated hard particles surrounded by a soft polymer shell. In the first part (Section 2), we provide details on sample preparation and characterization of the obtained materials. In a second part (Section 3), the viscoelastic behavior of the nanocomposites, characterized by dynamic mechanical measurements, is described. These experimental data illustrate the influence of filler-filler interactions on the linear mechanical behavior of these materials. At last (Section 4), different routes to model the (visco)elastic responses are discussed.

\section{Processing of nanocomposite systems}

A convenient way to process nanocomposite materials is based on the mixture of various aqueous suspensions (colloids) [9-12]. Emulsion polymerization is well known to provide in a simple way polymer colloidal suspensions with typical particle size in the range of ten to a few hundred nanometers. When both colloids are film forming, a co-continuous material can be expected, provided that the fraction of each component is large enough. Blending of hard and soft particles would lead, after film formation, to a random distribution of hard particles in the continuous soft matrix, with a certain probability to form aggregates depending on the volume fraction of filler. On the contrary, if the particles are structured, with a stiff core and a soft shell, then the material should consist, after shell coalescence, of a soft matrix with regularly dispersed stiff spherical domains, without contact between each other.

\subsection{Nanocomposites obtained from $P S-P B A$ and silica-PBA latex blends}

Different composites, based on the mixture of a film forming latex (matrix) with aqueous suspension of fillers in required proportions, were prepared. Choice was made of a poly(butyl acrylate) matrix (PBA, glass transition temperature $T_{\mathrm{g}}$ around $-47^{\circ} \mathrm{C}$ ) filled with spherical particles of either polystyrene (PS, $T_{\mathrm{g}}$ around $97^{\circ} \mathrm{C}$ ) or silica. Homopolymer latexes of PS or PBA were obtained through batch emulsion polymerization process. A typical recipe was as follows: deionized water $=900 \mathrm{~g}$, monomer $=90 \mathrm{~g}, \mathrm{NaHCO}_{3}$ $=0.75 \mathrm{~g}$, initiator $=0.75 \mathrm{~g}\left(\mathrm{~K}_{2} \mathrm{~S}_{2} \mathrm{O}_{8}\right.$ for PS and $\left(\mathrm{NH}_{4}\right)_{2} \mathrm{~S}_{2} \mathrm{O}_{8}$ for PBA), emulsifier $=2.91$ phm of NC12(3-(dimethyl dodecylammonium) propane-1-sulfonate) for PS, and $0.33 \mathrm{phm}$ of sodium dodecyl sulfate for PBA. The temperature of polymerization $=70^{\circ} \mathrm{C}$. Latexes were cleaned using ion exchange resins removing most of the surfactant, in order to avoid a possible effect on film forming process, film microstructure or film properties. They were cleaned in a non-diluted state (around $10 \mathrm{wt} . \%$ solid content) until conductivity remains constant. Particle sizes were around 110 $\mathrm{nm}$ for PS and $135 \mathrm{~nm}$ for the PBA latex (as measured by dynamic light scattering, Malvern Autosizer Lo-C instrument). Silica nanoparticles have been synthesized according to the Stöber process [13]. In a typical procedure, absolute ethanol (1555 g, Acros Organics), de-ionized water (24.9 g) and ammonia (12.75 M, 96.6 g, Laurylab) were filled into a $5 \mathrm{~L}$ polypropylene flask. The mixture was stirred at $160 \mathrm{rpm}$ to be homogenized and tetraethoxysilane (91.65 g, Fluka) was introduced at once. Reaction occurred at room temperature under continuous stirring for 2 weeks. The alcoholic silica suspension was dialyzed against water before use. Particle size was determined to be $125 \mathrm{~nm}$ by dynamic light scattering (DLS). The solid content of the resulting suspension was determined gravimetrically.

After blending different amounts of filler (PS or silica) (from 15 to 45 vol.\%) and PBA latexes, films were made by evaporation under controlled atmosphere during a delay long enough to achieve a complete maturation, i.e., 2 weeks at $35^{\circ} \mathrm{C}$ and $90 \%$ relative humidity. Although PBA matrix is transparent after maturation, highly filled composite films were rather opaque, suggesting aggregation of 


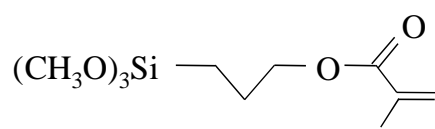

Fig. 1. Chemical formula of the 3-trimethoxysilyl propyl methacrylate silane coupling agent used in the silica encapsulation reaction.

the hard phase [14], as it has been previously observed on similar PS/PBA systems by small angle neutron scattering [15]. Films were tested after the film formation process (and called "as-dried films") or after a thermal treatment at $140^{\circ} \mathrm{C}$ for $4 \mathrm{~h}$.

\subsection{Nanocomposites obtained from encapsulated silica in $P(S-B A)$ copolymer}

The second nanocomposite system was based on encapsulated silica in a $\mathrm{P}(\mathrm{S}-\mathrm{BA})$ copolymer. The choice of this copolymer (with glass transition close to $0{ }^{\circ} \mathrm{C}$ was motivated by the possibility to test the mechanical behavior. As a matter of fact, we tried first with films issued from latex of encapsulated silica in PBA, but these films were very difficult to handle. One reason could be the formation of a high degree of crosslinking in these systems due to both chain transfer to polymer and bimolecular termination by recombination [16]. Such a mechanism is expected to significantly affect film formation and mechanical properties.

Since silica is initially hydrophilic, its surface needs to be modified to make possible anchoring and polymerization of the hydrophobic butyl acrylate and styrene (co)monomers through an emulsion polymerization process. This was achieved by the chemical grafting of an alkoxysilane bearing a polymerizable methacryloyl end group (see Fig. 1). The grafting was performed as described previously by direct addition of 3-trimethoxysilyl propyl methacrylate (MPS, Acros Organics) to an aqueous silica suspension [17]. The silica sol (30N50), with a mean hydrodynamic diameter of $68 \mathrm{~nm}$ as determined by DLS and $32 \mathrm{wt} . \%$ solid content, was kindly supplied by Clariant S.A. (France) and diluted in de-ionized water before use. A fixed amount of MPS (corresponding to $16 \mu \mathrm{mol} / \mathrm{m}^{2}$ silica surface) was introduced in the diluted silica sol $\left(10 \mathrm{~g} \mathrm{~L}^{-1}\right)$ containing $0.25 \mathrm{~g} \mathrm{~L}^{-1}$ sodium docedyl sulfate surfactant (SDS, Acros Organics). The reaction was conducted at room temperature for 1 week. The suspension was concentrated using an evaporating rotator before use.

The grafted silica particles were further engaged in a free radical polymerization process to make the polymer grow from their surface. The emulsion polymerization reaction was performed in batch at $70^{\circ} \mathrm{C}$ in a $250 \mathrm{~mL}$ double wall glass reactor fitted with a condenser. The reactor was charged with $100 \mathrm{~g}$ of the aqueous suspension containing the grafted silica beads $\left(37 \mathrm{~g} \mathrm{~L}^{-1}\right)$ and the surfactant (a mixture of SDS : $1.25 \mathrm{~g} \mathrm{~L}^{-1}$ and poly(oxyethylene) isooctyl cyclohexyl ether : TX-405, $0.75 \mathrm{~g} \mathrm{~L}^{-1}$, Aldrich). After degassing, the monomers from Aldrich (styrene : $36 \mathrm{gL}^{-1}$ and butyl

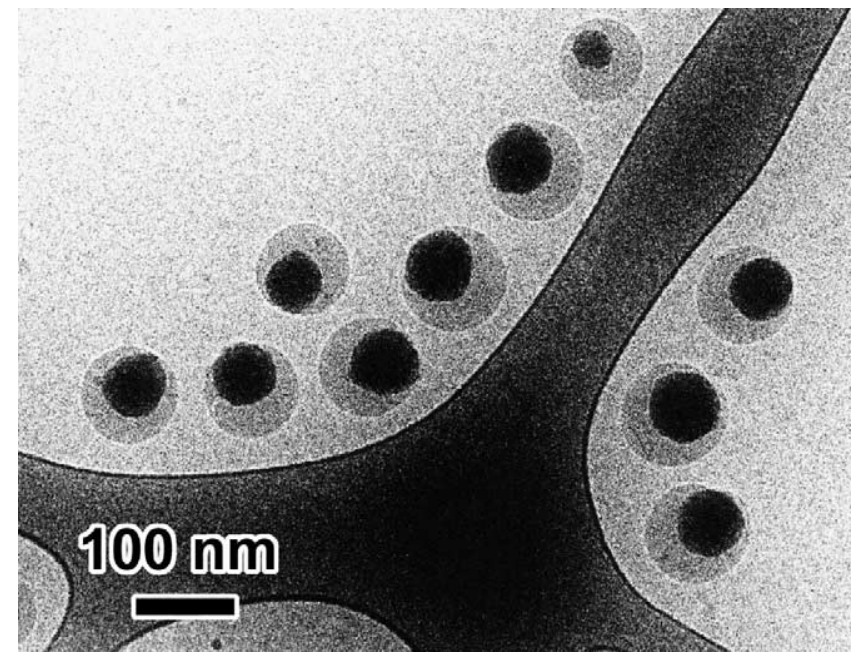

Fig. 2. Cryo-TEM image of grafted silica particles (dark) encapsulated with $\mathrm{P}(\mathrm{S}-\mathrm{BA})$ through emulsion polymerization.

acrylate : $63 \mathrm{~g} \mathrm{~L}^{-1}$ ) and the initiator (potassium persulfate : KPS, $0.5 \mathrm{~g} \mathrm{~L}^{-1}$, Acros Organics) were successively introduced at $70^{\circ} \mathrm{C}$ under stirring to start polymerization. The monomer to polymer conversion was of $86 \%$ as determined gravimetrically.

The morphology of the composite particles was characterized using cryo-transmission electron microscopy (cryo-TEM). Using the method described elsewhere [18,19], thin liquid films of the particle suspension were formed on carbon membranes and rapidly frozen into liquid ethane. The particles were then observed at low temperature embedded in a preserving film of vitreous ice, using a Philips CM200 'Cryo' microscope operated at an accelerating voltage of $80 \mathrm{kV}$. The TEM image in Fig. 2 clearly attests for a successful encapsulation of the nanometric silica particles by the $\mathrm{P}(\mathrm{S}-\mathrm{BA})$ copolymer. Although the silica beads appear to be uncentered in the polymer shell, they are expected to be homogeneously distributed in the coalesced latex film. However, a limited number of silica/silica contacts may be present.

For comparison purpose, blends of silica and $\mathrm{P}(\mathrm{S}-\mathrm{BA})$ copolymer latexes were prepared by mixing together the silica sol and a latex of P(S-BA). This copolymer latex was obtained from the previous latex of encapsulated silica in $\mathrm{P}(\mathrm{S}-\mathrm{BA})$, after having eliminated the grafted silica by centrifugation. Silica/P(S-BA) nanocomposites were obtained after maturation of latex blends or encapsulated latex during 2 weeks at $35^{\circ} \mathrm{C}$ and $90 \%$ relative humidity.

\section{Viscoelastic behavior}

The dynamic shear moduli $\left(G^{\prime}, G^{\prime \prime}\right)$ of various nanocomposites were measured as a function of temperature with a homemade inverted pendulum working in helium atmosphere [20]. Experiments were performed in the temperature 

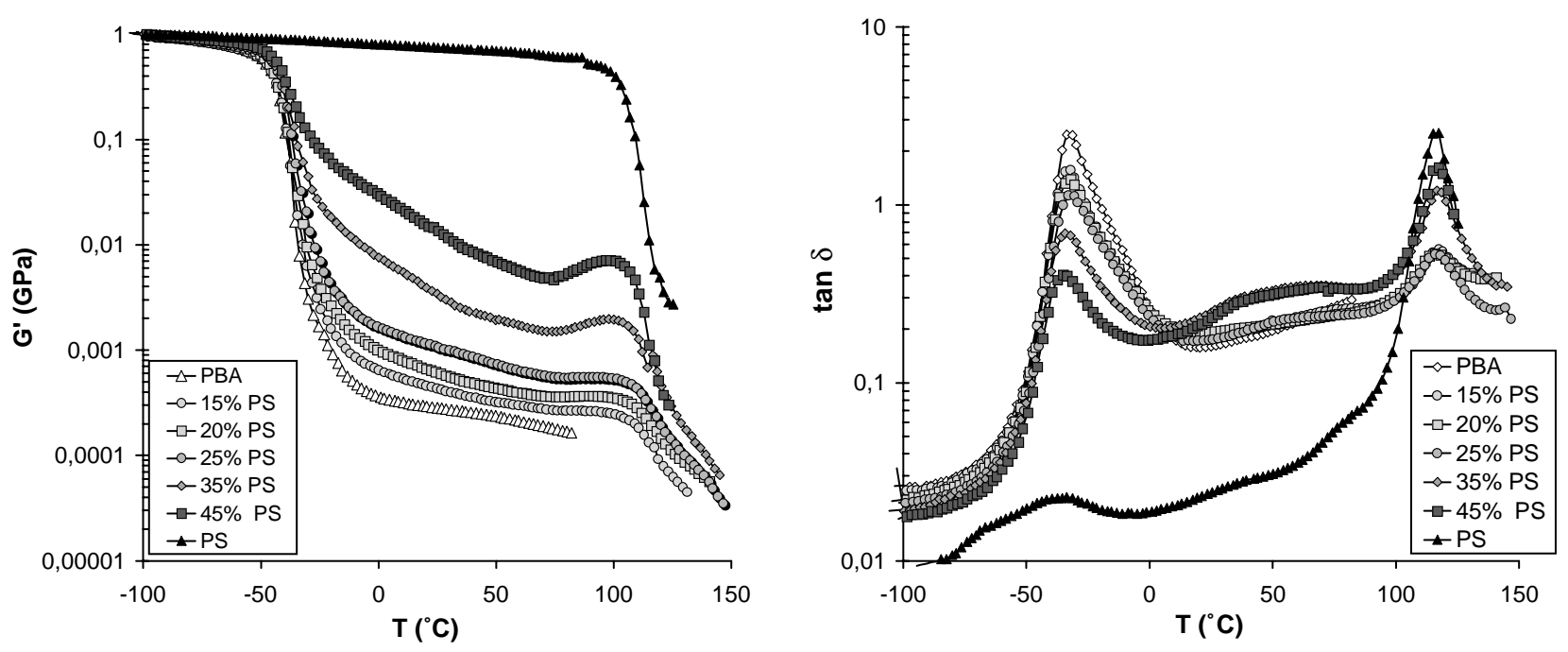

Fig. 3. Real shear modulus and loss factor vs. temperature for "as dried" PS-PBA nanocomposites with different filler amounts (1 Hz).

range $\left[-170\right.$ to $\left.150{ }^{\circ} \mathrm{C}\right]$ with a heating rate of $1{ }^{\circ} \mathrm{C} / \mathrm{min}$ and at a fixed frequency of 0.1 or $1 \mathrm{~Hz}$.

\subsection{PS-PBA nanocomposites}

The dynamic mechanical responses of PBA and PS/PBA "as-dried systems" (i.e., just after PBA maturation) are shown in Fig. 3. The properties of a film of pure PS (obtained by freeze-drying/hot pressing) are also reported. A significant reinforcement of the shear modulus is observed in between PS and PBA main (or $\alpha$ ) relaxations $\left(-32^{\circ} \mathrm{C}\right.$ $\leq T \leq 118^{\circ} \mathrm{C}$ ). One can notice that this reinforcement is not linear: the faster increase is observed around $20 \mathrm{vol} . \%$, i.e., near the geometric percolation threshold. In the same temperature range, the height and the width of the $\tan \delta$ peak (associated with the PBA glass transition) decrease, with a slight shift of the relaxation to lower temperature. Nevertheless, differential scanning calorimetry measure- ments performed on these systems showed that the PBA glass transition temperature remains constant and equal to $-47^{\circ} \mathrm{C}$ (for a heating rate of $10^{\circ} \mathrm{C} / \mathrm{min}$ ) whatever the filler content. Hence, the shift of $T_{\alpha}$ to lower temperature as the filler content increases is probably due to a mechanical coupling effect. In addition to a higher level of the relaxed modulus, the temperature dependence of composite moduli differs from that observed for the pure matrix and for the filler phase. For the composite materials, the relaxed modulus first decreases until $70^{\circ} \mathrm{C}$ and then increases before falling again above the PS main relaxation. These two counteracting phenomena lead to the presence of a bump in the the $\tan \delta$ curves, located between the peaks associated with the PBA and PS main relaxations. As discussed in [21], these two phenomena are a consequence of the evolution of filler-filler interactions with temperature: in the low temperature range $\left(T \leq 70^{\circ} \mathrm{C}\right)$, the decrease of relaxed composite moduli can be attributed to a decrease of
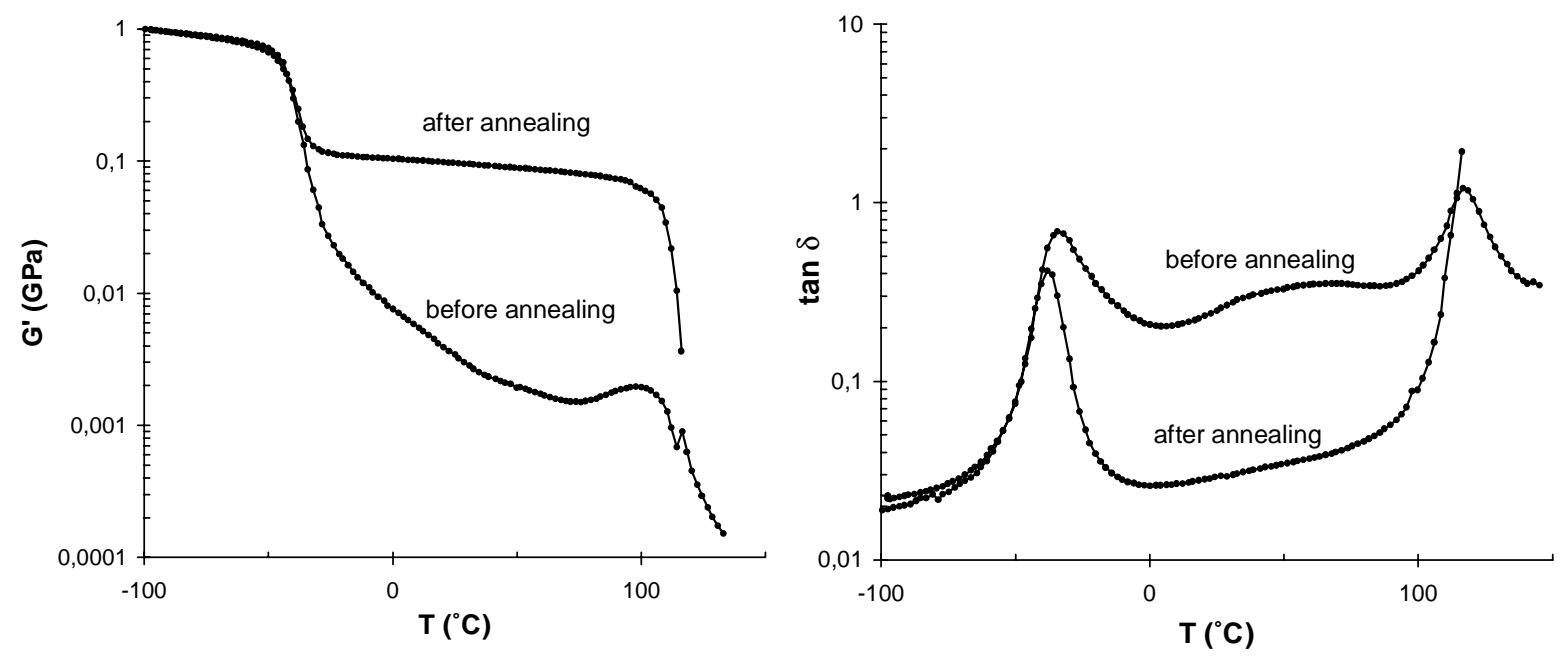

Fig. 4. Real shear modulus and loss factor for PBA samples filled with 35\% PS, before and after thermal treatment $(1 \mathrm{~Hz})$. 

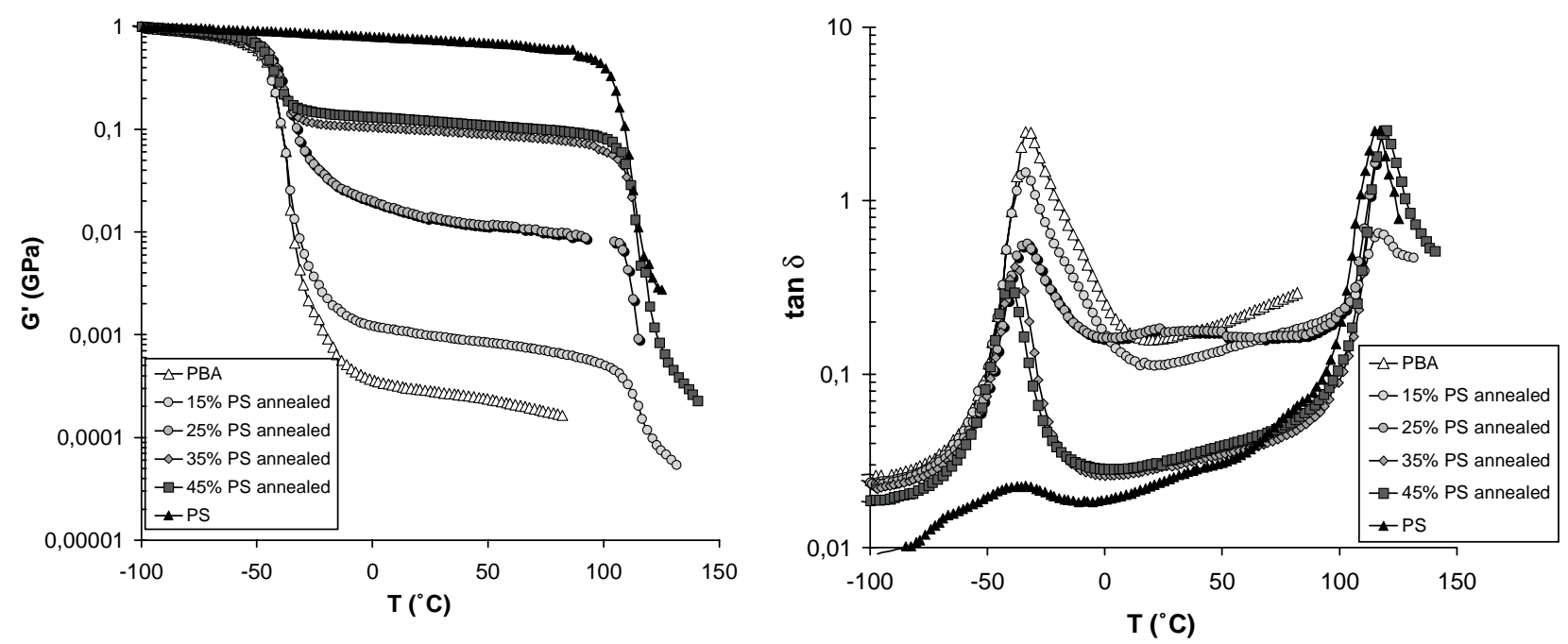

Fig. 5. Real shear modulus and loss factor vs. temperature for "annealed" PS-PBA blends with different filler amounts $(1 \mathrm{~Hz})$.

filler-filler interactions via an immobilized matrix, whereas above $70^{\circ} \mathrm{C}$ the increase of composite moduli should come from the very beginning of the coalescence between PS particles leading to an enhancement of filler-filler interactions. The formation of a rigid network starts to occur but, as temperature approaches the PS glass transition, PS aggregates soften until complete transformation into rubbery behavior.

\subsubsection{Annealing effects}

Similar tests were performed on PS filled composites heated at $140^{\circ} \mathrm{C}$ (well above the PS glass transition) during $4 \mathrm{~h}$, to achieve the complete coalescence of neighboring PS particles. Fig. 4 shows $G^{\prime}$ and $\tan \delta$ versus temperature for sample filled with $35 \%$ PS before and after the thermal treatment. The annealing treatment leads to a higher relaxed modulus and a lower value of $\tan \delta$ between the two relax- ations. Moreover, the decrease of $G^{\prime}$ with temperature follows an evolution similar to that measured for pure PS. This indicates that the temperature dependence of the modulus is now governed by the stiff connected phase (PS). The very low value of the loss factor can be attributed to the presence of a rigid network preventing particle friction. Similar experiments performed with other filler concentrations are reported in Fig. 5. After thermal treatment, the increase of the rubbery plateau is observed even for the lowest filler volume fraction $(15 \%)$. That indicates that particle aggregation may lead to an increase of the shape factor, and consequently has an effect on reinforcement even below the percolation threshold. These results obtained for "as-dried" and annealed samples underline the differences in mechanical responses between a system in which the particles are just in contact and a system in which the particles are strongly bonded.
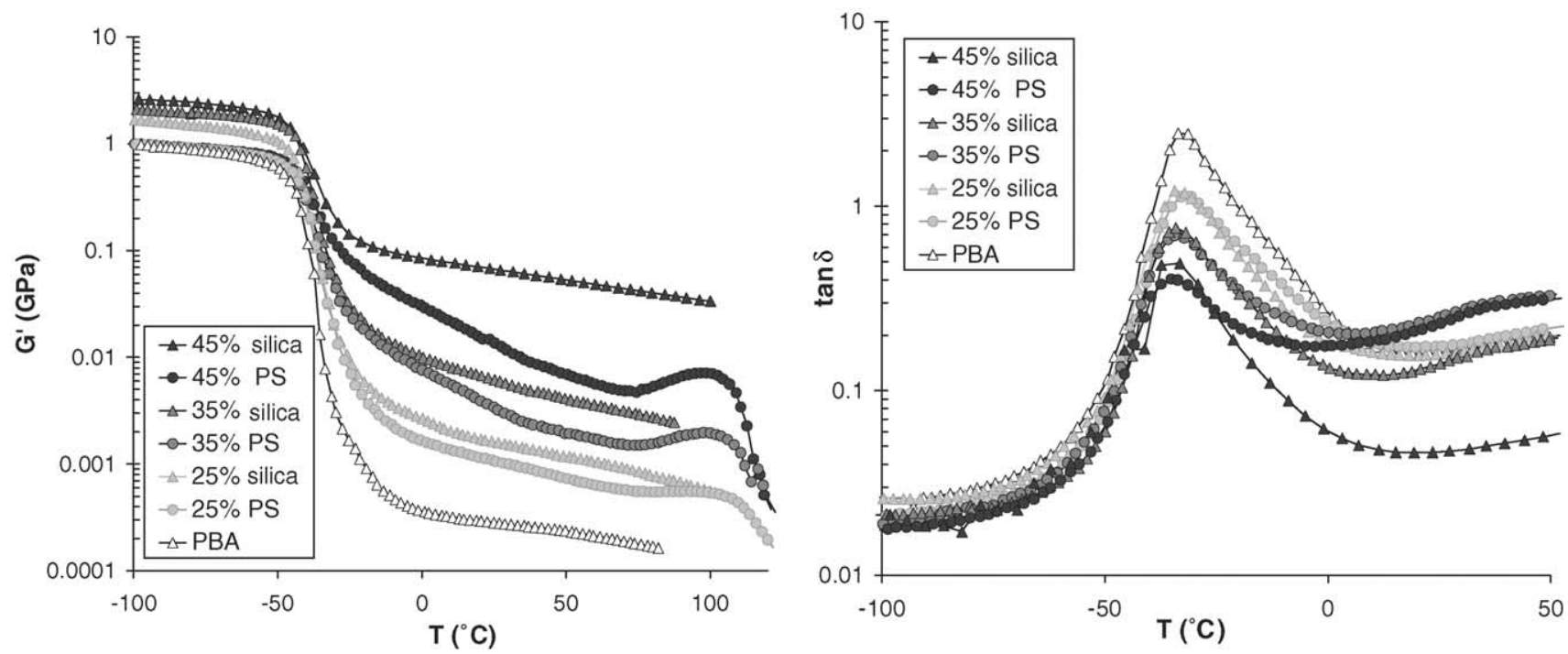

Fig. 6. Real shear modulus and loss factor vs. temperature $(1 \mathrm{~Hz})$ for PBA samples filled with PS $(\bigcirc)$ compared to silica filled nanocomposites $(\triangle)$. 

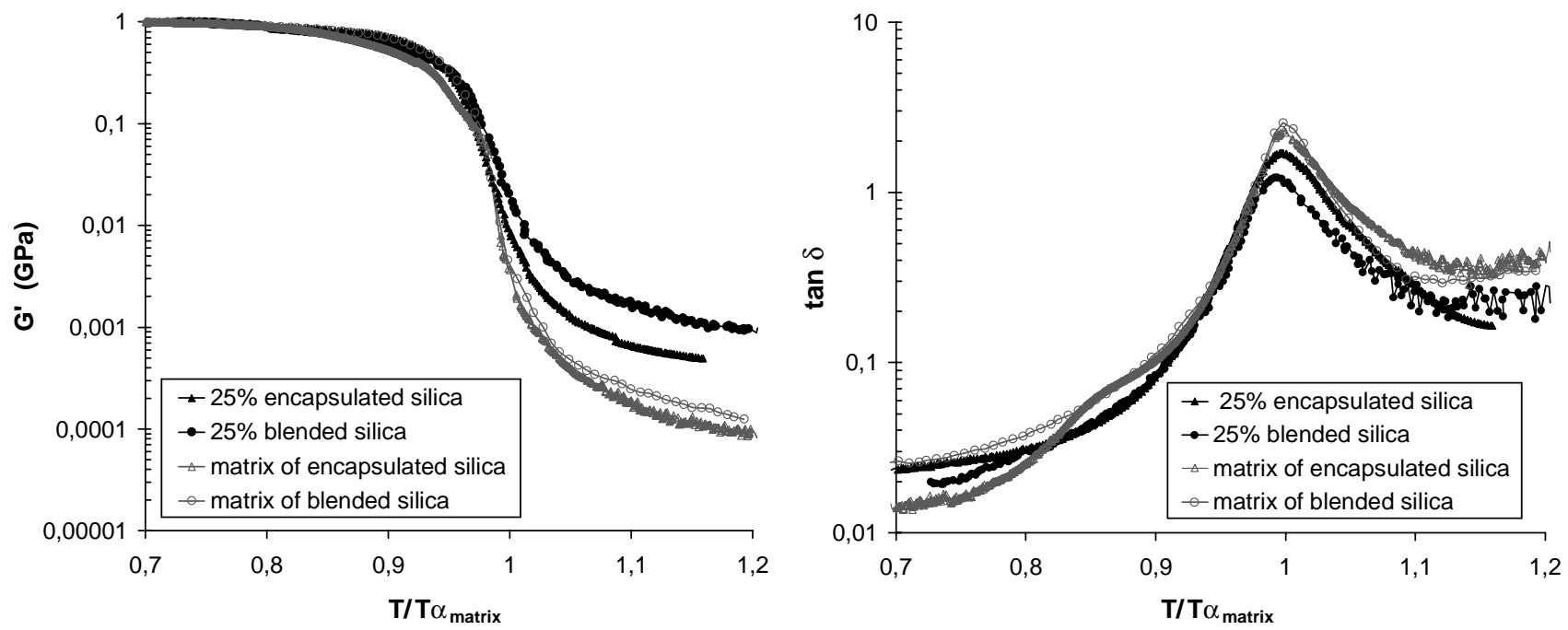

Fig. 7. Real shear modulus and loss factor vs. normalized temperature for $\mathrm{P}(\mathrm{S}-\mathrm{BA})$ samples filled with $25 \%$ silica $(0.1 \mathrm{~Hz})$ : $(\mathrm{O})$ film obtained from blend of silica and matrix suspensions, $(\triangle)$ film obtained from core(silica)/shell(matrix) latex.

\subsection{Comparison with silica-PBA nanocomposites}

The dynamic mechanical spectrometric responses of PBA-silica filled composites are compared with those of PBA-PS composites in Fig. 6. Two interesting points can be stressed: (1) In the glassy state, a slight reinforcement is observed for silica fillers (whose shear modulus is about thirty times higher than that of the glassy matrix), as expected from a simple mixture effect. On the contrary, no reinforcement is observed in the glassy state in case of PS-PBA systems since both phases have similar moduli. (2) In the rubbery plateau, composites filled with silica particles exhibit a higher modulus and a weaker temperature dependence than those filled with PS particles. These results suggest once more that the strength of filler-filler interactions (Hydrogen bonds between silica particles versus weaker Van der Waals bonds between PS particles) has an effect on the level of the relaxed modulus and the temperature dependence.

\subsection{Silica-P( $S-B A)$ nanocomposites}

The mechanical study was extended to $\mathrm{P}(\mathrm{S}-\mathrm{BA})$ films filled with 25\% silica. In Fig. 7 the viscoelastic behavior of nanocomposites made from a mixture of silica and $\mathrm{P}(\mathrm{S}-\mathrm{BA})$ suspensions is compared with that of a nanocomposite fabricated from a core (silica)/shell $\mathrm{P}(\mathrm{S}-\mathrm{BA})$ latex. In this figure, temperatures are normalized in order to take into account the shift of temperature of main relaxation observed between the two $\mathrm{P}(\mathrm{S}-\mathrm{BA})$ matrices $\left(8^{\circ} \mathrm{C}\right)$, despite precautions that have been taken to have the same matrix in both films. These experiments show clearly that the reinforcement in the rubbery state decreases when the short range interactions between fillers are decreased. All these results, which show very different responses of well controlled materi- als and morphologies, are a challenge for micro-mechanical modeling.

\section{Modeling approaches}

Such approaches aim at modeling the mechanical interactions between the constituents of the considered composite materials so as to reproduce the main experimental observations. As a first step, this can be done in a simple way by using phenomenological models, such as the serial-parallel model of Takayanagi [22], which does not take explicitly into account any morphological characteristics but gives a direct uniaxial representation of the presumed mechanical coupling through adequate serial or parallel connections and some adjustable parameters. The serial-parallel model has been extended by taking into account the percolation concept $[23,24]$. In that case, the fraction of filler acting in parallel, corresponding to the percolated network of filler, is derived from percolation theory. It depends on two parameters, namely the percolation threshold $\left(f_{\mathrm{c}}^{\mathrm{i}}\right)$ and the critical exponent $(t)$. Fig. 8 a shows the evolution of the shear modulus predicted by this model, calculated with parameters given in [24], i.e. a percolation threshold $f_{\mathrm{c}}^{\mathrm{i}}=0.154$ for spheres, and a critical exponent $t=1.8$. Such a model accounts well for the reinforcement in case of strong filler interactions (annealed PS-PBA systems) whereas it is too stiff for weaker filler interactions (as-dried PS-PBA systems) (see Fig. 8a). In this figure and as for the other modelings realized in this section, the reinforcement obtained at $60^{\circ} \mathrm{C}$ has been considered, supposing that filler-filler interactions via immobilized matrix were lower at this temperature [21] (case of as-dried PS/PBA nanocomposites).

More predictive treatments can be developed in the framework of homogenization theory by proposing an explicit 

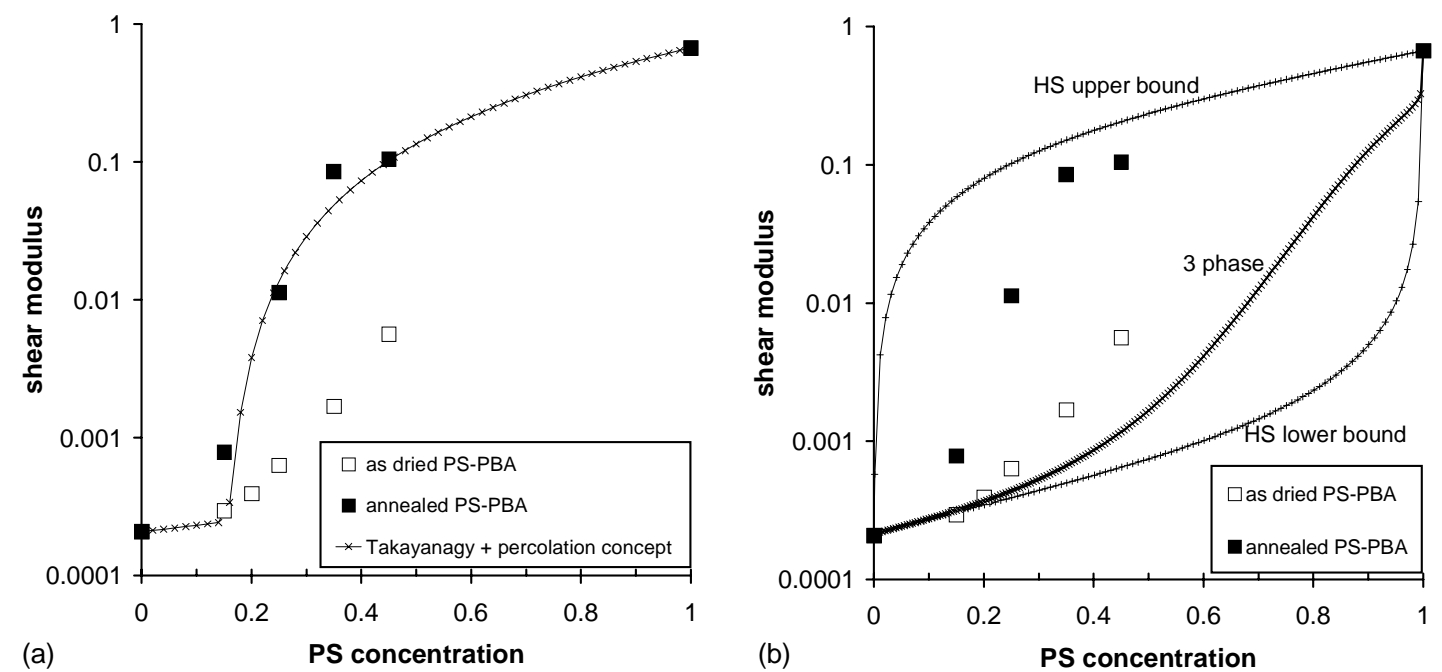

Fig. 8. Reinforcement at $60^{\circ} \mathrm{C}$ vs. volume fraction of filler for PS-PBA nanocomposites : ( $\square$ ) as-dried, ( $\square$ ) after thermal treatment $\left(4 \mathrm{~h}\right.$ at $\left.140^{\circ} \mathrm{C}\right)$. (a) Percolation approach with percolation exponent $=1.8$ and geometrical percolation threshold $=0.154$, (b) Generalized ( 3 phase) self-consistent estimation, classical HS bounds and experimental data.

connection between given morphological informations on the microstructure of a "representative volume element" (RVE) of the considered material and the overall mechanical response of the associated "equivalent homogeneous medium" (EHM). The classical way to describe the morphology is to use point correlation functions (of increasing order) of the local moduli. Bounds for the overall quantities can then be derived from variational approaches, when available: this is possible for elasticity or, for specific loading paths such as those used above (DMA), and also for linear viscoelasticity. However, in the case of polymer based composites, there is a huge difference between filler and polymer moduli (e.g., shear modulus of silica $\approx 30 \mathrm{GPa}$, glassy polymer $\approx 1 \mathrm{GPa}$, rubber $\approx 1 \mathrm{MPa}$ ), so that the obtained bounds (of first, second and third order using, respectively, first, second and third-order correlation functions) are gen- erally too far apart to be of practical use for comparison with experimental data (as illustrated for Hashin-Shtrikman bounds in Fig. 8b). In addition, point correlation functions are not a very convenient tool to express, as wished here, the connectivity of a given phase, which would need correlation functions of very high order, out of reasonable reach from experiment.

An alternative method is related to the so-called "morphologically representative pattern-based approach" (MRP) [25], which describes the microstructure with use of finite composite patterns instead of points; the material content of such patterns, which are mapping the whole RVE, is supposed to be known as well as some statistical characteristics of their spatial distribution. Several typical morphologies can be modeled in this way, such as the matrix/inclusion situations with isolated particles in a continuous matrix, and vari-
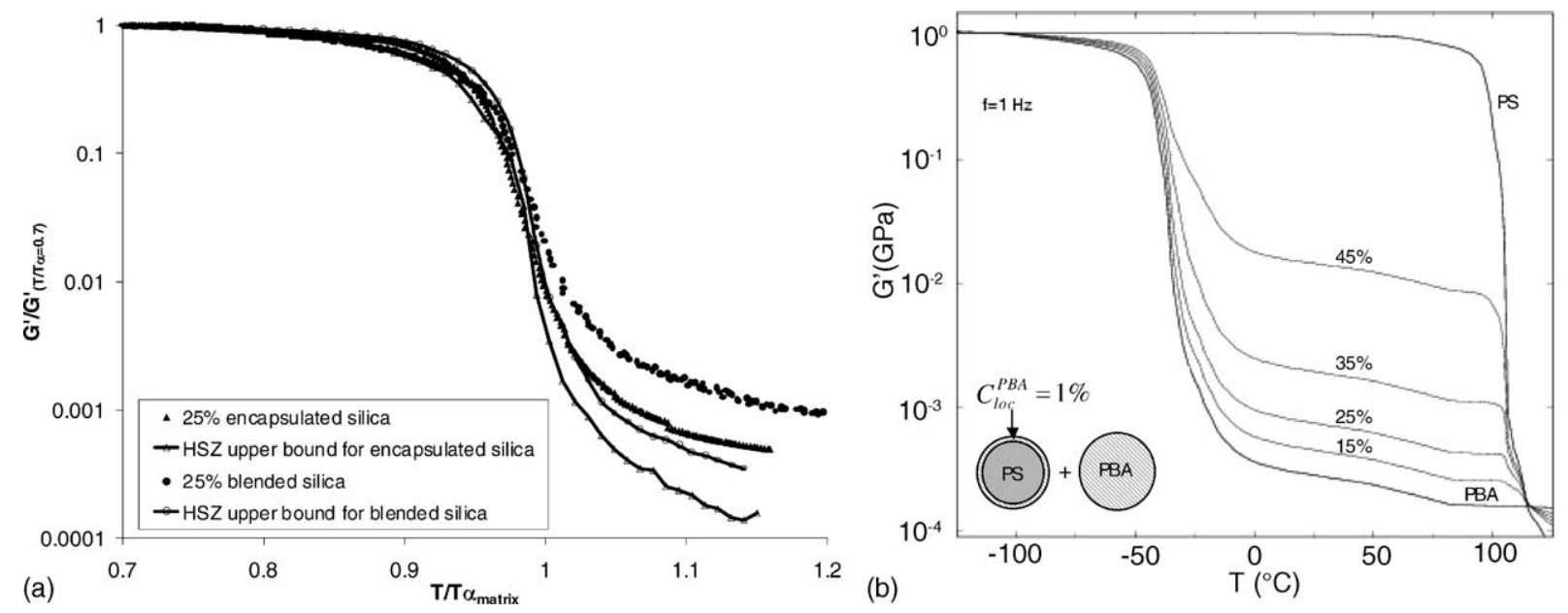

Fig. 9. (a) P(S-BA) samples filled with $25 \%$ silica $(0.1 \mathrm{~Hz})$, comparison between experimental data and the upper HSZ bound. (b) Self-consistent estimates for the real shear moduli of PS/PBA nanocomposites using two MRPs. 
ational methods, similar to those related to classical "point" approaches, can be developed. Since the corresponding morphological information is richer, the resulting bounds are sharper. For instance, the real shear moduli of silica/P(S-BA) nanocomposites are compared in Fig. 9a to the upper bound for particulate composites described by the Hashin composite sphere assemblage (the so-called HSZ bound [26]). Since experimental data lie above the bound, even those for the nanocomposite issued from encapsulated silica, a third phase of additional chemical agent used for the process (non grafted MPS) or of immobilized matrix might play a role.

Estimates can also be derived from specific adequate assumptions, both for elastic and viscoelastic moduli. When applied to the Hashin composite sphere (or cylinder) assemblage, former models can be recovered (e.g., Kerner's model [27], the 3-phase model-Christensen and Lo's [28]—or the $n$-phase model-Hervé and Zaoui [29]). The latter generalized self-consistent estimate is obtained by an iteration technique, embedding the different phases or more specific patterns in the EHM itself [30]. Three-phase predictions are plotted in Fig. $8 \mathrm{~b}$ and compared to Hashin-Shtrikman bounds [31] and the experimental data. This treatment is satisfactory when particles are well dispersed in the matrix and far enough apart from each other. However, as soon as particle aggregation occurs and especially when the rubbery plateau modulus is concerned, the 3-phase model yields far too soft predictions. Improvements of this treatment can be developed in order to better take into account aggregation effects by combining, as authorized by the MRP theory, different elementary patterns, as in [32]. For instance, Fig. 9 shows estimates calculated using two patterns, namely a stiff core in a thin soft shell and a soft shell, that makes possible to account for locally higher concentration of the filler phase. Another possibility is to embed the particles in an additional shell of stiffer matrix which could represent that part of the polymer matrix with reduced molecular mobility which is blocked within particles [21].

To go further, two different treatments have been investigated: both of them focus on the mechanical effects of contact between neighboring particles. The first one (Section 4.1) still refers to the MRP approach whereas the second one (Section 4.2) makes recourse to a discrete simulation.

\subsection{MRP approach}

The patterns were defined in order to enhance the continuity of the filler phase while the matrix still remains connected. Two internal pattern contents were tested: inside a spherical shell of matrix phase, the filler phase occupies either a cylinder or a disc touching the pattern border. The cylinder radius and the disc thickness depend on the filler concentration (Fig. 10). In the assemblage, the orientation $\theta$ of the disc or cylinder is assumed equiprobable and the spatial distribution of the centers of the patterns is isotropic. The self-consistent estimate of the effective elastic properties $\left(C^{\text {eff }}\right)$ of such materials is obtained from the FEM computation of the mechanical response of a MRP embedded in an infinite medium with moduli $\left(C^{\text {eff }}\right)$ and subjected to homogeneous strain conditions at infinity.

This localization problem has been solved for all filler inclusions oriented with an angle $\theta=0$. Due to the MRP axisymmetric geometry, the average localization tensors $A_{\theta=0}^{\mathrm{r}}$ of each phase $r$ (matrix or inclusion in the pattern with the orientation $\theta=0$ ) exhibit transversely isotropic symmetry. They are characterized by six components that are obtained by the computation of the average stress and strain tensors in the composite inclusion for four applied strain conditions (longitudinal and transverse shear, plane compression and

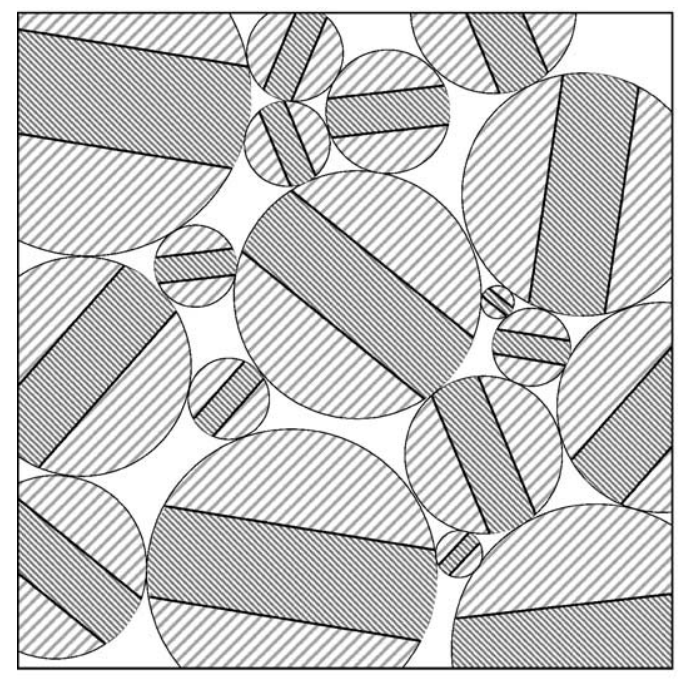

(a)
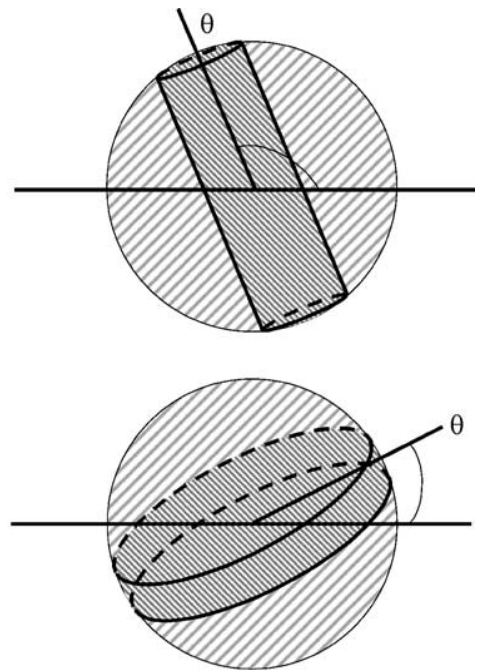

(b)

Fig. 10. (a) Arrangement of spherical "morphologically representative patterns". (b) Internal structure of patterns: the filler phase occupies either a cylinder or a disc. Contacts between fillers are possible in the arrangement. 
uniaxial load). These calculations have been conducted in 2D FEM models using Fourier and axisymmetric modes. For these computations, the radius of the spherical "infinite" medium was four times larger than the radius of the composite inclusion (as shown by other calculations, a ratio between the two radii larger than 3 is sufficient [30]), the number of eight-noded elements was between 700 and 900, the degrees of freedom around 5000 [33].

In principle, one should solve the localization problems for all orientations $\theta$ and compute the average tensors $\left\langle A_{\theta}^{\mathrm{r}}\right\rangle_{\theta}$; the self-consistent estimate $C^{\text {eff }}$ is then given by:

$C^{\mathrm{eff}}=C^{\mathrm{m}}+f^{\mathrm{i}}\left(C^{\mathrm{i}}-C^{\mathrm{m}}\right):\left\langle A_{\theta}^{\mathrm{i}}\right\rangle_{\theta}$

where the superscripts $\mathrm{i}$ and $\mathrm{m}$ refer to the inclusion and matrix, respectively and $f^{i}$ is the filler concentration. However, the recourse to isotropy invariants allows one to compute $\left\langle A_{\theta}^{\mathrm{i}}\right\rangle_{\theta}$ from $A_{\theta=0}^{\mathrm{i}}$ only, which makes the computation easier. The implicit self-consistent equation given above $\left(A_{\theta=0}^{\mathrm{i}}\right.$ depends on $C^{\text {eff }}$ ) is solved iteratively by means of a simple fixed point algorithm. Detailed equations are given in [33]. With a convergence criterion of the order of $10^{-5}$, depending on the morphology of the pattern, 5 to 60 iterations were necessary to obtain the self-consistent estimate.

Fig. 11 compares the self-consistent estimates for the shear modulus of the composite for filler inclusions being either cylinders, discs, spheres (this third case corresponds to the classical " 3 -phase" model where only the matrix is continuous). One can observe that the use of cylinders and discs leads to the appearance of a quasi-mechanical percolation located around 30\% and 50\%, respectively. These percolation thresholds are governed by the surface fraction of emerging filler within the MRP, which depends itself on the filler inclusion shape. For the 3-phase model, no percolation of the filler phase is observed. Thus, the use of adequate MRP seems to be a promising approach for modeling mechanical percolation through the self-consistent type homog-

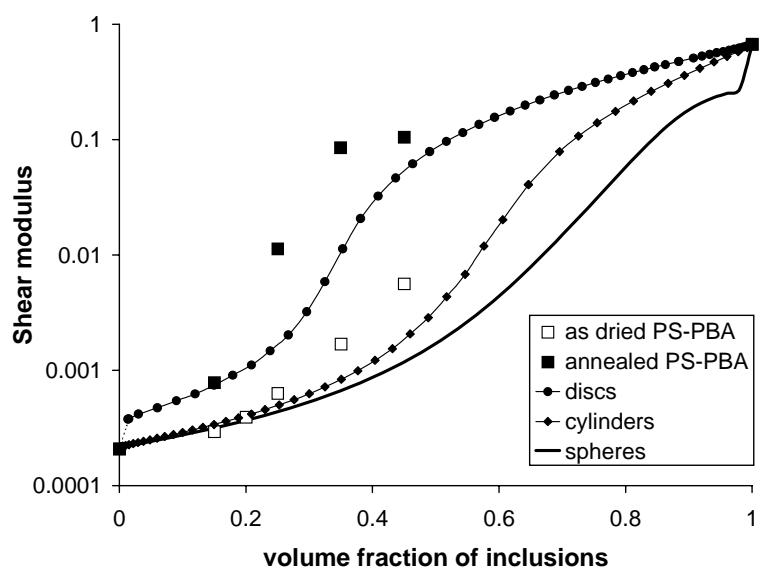

Fig. 11. Shear modulus of composites with inclusions being either cylinders, discs or spheres. For the computation, the matrix and filler phases have the properties of PBA $(K=1 \mathrm{GPa} ; G=2 \mathrm{MPa})$ and PS $(K=2.4 \mathrm{GPa} ; G=669 \mathrm{MPa})$ at $60^{\circ} \mathrm{C}$. enization technique. Further improvements of this technique could be obtained by using various MRPs, with more realistic filler geometries and accounting for the actual statistics of contacts between fillers. As a whole, this method offers the advantage of dealing with different possible morphologies of particles and of predicting some moderate mechanical percolation effects for adequate pattern contents. Nevertheless, it suffers from the fact that only averaged pair interactions between patterns far away from each other can be taken into account through their interactions with the effective medium, while pair interaction between first neighbor patterns are smoothed out in a way that might not be an accurate description of their actual contribution to the effective behavior. Any improvement would depend on a more detailed description of the pattern contents, involving several fillers in close interaction in various configurations, and this could make the numerical computations more difficult. That is the reason why a different approach has been developed too, in order to enhance the influence of short range interactions between fillers through a discrete treatment.

\subsection{Discrete simulation}

Another attempt for a better understanding of elastic properties of nanocomposites is to focus on the short range interactions between filler particles and between matrix and filler. In this scope, a discrete numerical model taking explicitly into account the microstructure and the nature of contacts between phases has been developed [21,34]. Calculations are made on a close-packing of hard and soft monodisperse spheres (e.g. Jagota [35]), which represent either the filler particles or the matrix particles $(\approx 1000$ spheres with a packing density $\approx 0.57$ ). At each contact between neighbouring spheres, three forces and three moments are transmitted. These efforts are expressed as a linear function of the displacements and the rotations of the connected spheres, exhibiting different "contact stiffnesses" (normal, tangential, flexural and torsional) which depend both on the modulus of each connected sphere and on the contact area, characterized by the parameter $\alpha=\left(R_{\mathrm{c}} / R\right)^{2}$, where $R_{\mathrm{c}}$ and $R$ are the contact radius and the sphere radius, respectively. The larger the contact area (i.e. $\alpha$ ), the higher the transmission of moments through the contact. Three kinds of contacts were considered: soft/soft, hard/hard and soft/hard contacts. As the matrix is continuous and totally wets the filler particles, the contact area radius of soft/soft and hard/soft contacts is approximated by the radius of the spheres themselves. For hard/hard contacts, we used a variable contact area (i.e. $\alpha^{\text {hh }}$ ) in order to quantify the effect of the strength of filler-filler interactions on the effective modulus which is numerically deduced by applying adequate boundary conditions.

Fig. 12 shows the effect of the strength of hard/hard contact (i. e. $\alpha^{\text {hh }}$ ) on the evolution of the packing modulus with the filler ratio. When filler-filler interactions are stronger $\left(\alpha^{\text {hh }}=1\right)$, the effective modulus increases suddenly at the geometrical percolation threshold $\phi_{\mathrm{c}}=18.5 \mathrm{vol} . \%$. When 


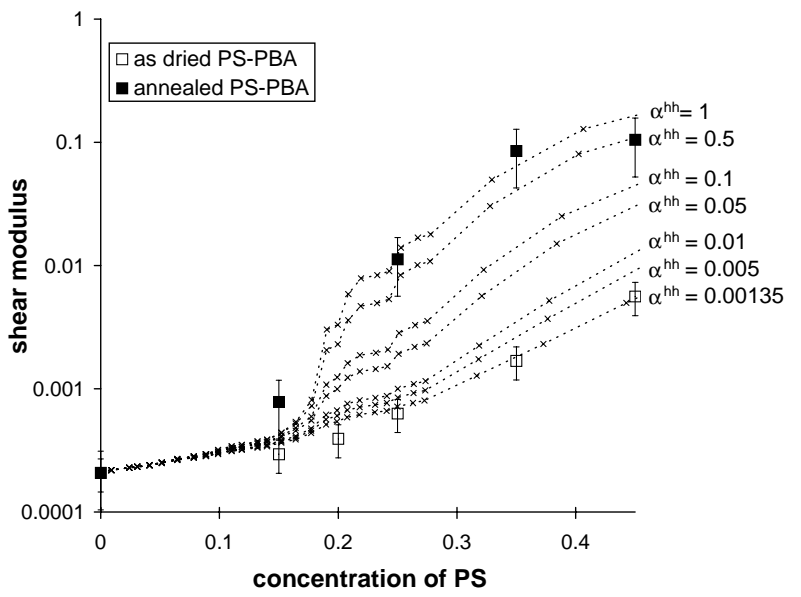

Fig. 12. Real shear modulus of PS-PBA nanocomposites at $60^{\circ} \mathrm{C}$ before $(\square)$ and after ( $\square$ ) the annealing treatment. (-x-) Modelling with the discrete numerical simulation varying the strength of filler-filler interactions $\left(\alpha^{\mathrm{hh}}\right)$.

filler-filler interactions are weaker $\left(\alpha^{\text {hh }} \leq 10^{-2}\right)$, i.e., when hard particles are allowed to roll on each other, there is no discontinuity at the percolation threshold. The weaker the strength of filler-filler interactions, the lower is the packing modulus. Experimental values obtained for PS-PBA nanocomposites at $60^{\circ} \mathrm{C}$ in "as-dried" and "annealed" states are also reported in Fig. 12. After the annealing treatment, PS particles are strongly bonded. As a consequence, calculations made with $\alpha^{\mathrm{hh}}=1$ (forces and moments are transmitted as in bulk PS) are in very good agreement with experimental data. Before the annealing treatment, the strength of filler-filler interaction is weaker and the value $\alpha^{\text {hh }}=$ $1.35 \times 10^{-2}$, determined by inverse method, allows one to account very well for all PS volume fractions. The same approach could be used for modeling the whole temperature evolution of "as-dried" composite moduli. However, adjusting a new value of the contact area at each temperature is not quite satisfactory. In the detailed paper [21] a physical law describing the temperature evolution of the contact area is proposed.

\section{Conclusion}

As a conclusion, the study of various model systems, which makes possible to vary the contrast between filler and matrix, the filler ratio and the short range interactions between filler particles, has shown that the reinforcement increases when the filler ratio increases and when the strength of filler interactions increases. In case of strong short range filler interactions (for instance chemical bonds between PS particles after the annealing treatment), the relaxed modulus increases significantly above the geometric percolation threshold. This can be attributed to the formation of a quasi-rigid network. In case of weak filler interactions (for instance PS-PBA systems before the annealing treatment), the relaxed modulus decreases faster with temperature when PS concentration increases, suggesting a temperature dependence of filler interactions. Such a temperature dependence may not be invoked for silica-PBA systems and PS-PBA annealed systems. At last, it has been shown that the decrease of filler interactions thanks to an encapsulation process leads to a lower reinforcement. The challenge of modeling these various responses by micromechanical approaches has only been partly answered at the moment. The most promising treatments refer to the introduction of refined statistical information on the contacts between fillers in the MRP approach on the one hand and to the development of a physically sound modeling of the rate and temperature dependence of the contact interactions in the discrete simulation on the other hand.

\section{Acknowledgements}

This work was performed under the auspices of "Programme Matériaux" from CNRS under contract no. 113. One of the authors (E. Chabert) was supported through the Brite-Euram project BE 97-4448 administrated by the $\mathrm{Eu}-$ ropean Community. C. Graillat is also gratefully acknowledged for providing technical help.

\section{References}

[1] R. Rothon, M. Hancock, Particulate-filled polymer composites, R. Rothon, Essex, Longman Scientific and Technical, 1995.

[2] M. Sumita, T. Shizuma, K. Miyasaka, K. Ishikawa, Macromol. Sci. Pol. Phys. B22 (1983) 601.

[3] E.P. Giannelis, R. Krishnamoorti, E. Manias, Ad. Polym. Sci. 138 (1999) 107.

[4] P.C. LeBaron, Z. Wang, T.J. Pinnavaia, Appl. Clay Sci. 15 (1-2) (1999) 11

[5] K. Varlot, E. Reynaud, M.H. Kloppfer, G. Vigier, J. Varlet, J. Polym. Sci. B39 (2001) 1360.

[6] L. Chazeau, C. Gauthier, G. Vigier, J.-Y. Cavaillé, Relationships between microstructural aspects and mechanical properties of polymer-based nanocomposites, in: H.S. Nalwa (Ed.), Handbook of Organic-Inorganic Hybrid Materials and Nanocomposites, vol. 2, American Scientific Publishers, 2003.

[7] V. Favier, R. Dendievel, G. Canova, J.-Y. Cavaillé, P. Gilormini, Acta Mater. 45 (1997) 1557.

[8] Z. Hashin, J. Appl. Mech. 50 (1983) 481.

[9] J.-Y. Cavaillé, R. Vassoile, G. Thollet, L. Rios, C. Pichot, Colloid Polym. Sci. 269 (1991) 248.

[10] M. Hidalgo, thesis, INSA-Lyon, France, 1991.

[11] S.T. Eckersley, B.J. Helmer, J. Coat. Technol. 69 (1997) 864.

[12] N. Agarwal, R. Farris, J. Appl. Polym. Sci. 72 (11) (1999) 1407.

[13] W. Stöber, A Fink, E. Bohn, J. Colloid Interf. Sci. 26 (1968) 62.

[14] J. Feng, M.A. Winnik, R. Shivers, Macromolecules 28 (1995) 7671.

[15] Y. Chevalier, M. Hidalgo, J.-Y. Cavaillé, Macromolecules 32 (1999) 7887.

[16] A. Ghielmi, G. Storti, M. Morbidelli, Chem. Eng. Sci. 56 (2001) 937.

[17] E. Bourgeat-Lami, J.L. Luna-Xavier, A. Guyot, in: R.M. Laine, C. Sanchez, C.J. Brinker, E. Gianellis (Eds.), Organic/Inorganic Hybrid 
Materials, Mat. Res. Soc. Symp. Proc., vol. 628, San Francisco, 2000, CC3.5.1.

[18] J.-L. Putaux, A. Buléon, R. Borsali, H. Chanzy, Int. J. Biol. Macromol. 26 (1999) 145.

[19] S. Chalaye, E. Bourgeat-Lami, J.-L. Putaux, J. Lang, Macromol. Symp. 169 (2001) 89.

[20] J.Y. Cavaillé, M. Salvia, P. Merzeau, Spectra 2000 (16) (1988) 37.

[21] E. Chabert, R. Dendievel, C. Gauthier, J.-Y. Cavaillé, Compos. Sci. Technol. 64 (2004) 309.

[22] M. Takayanagi, S. Uemura, S. Minami, J. Polym. Sci. C 5 (1964) 113.

[23] N. Ouali, J.-Y. Cavaillé, J. Perez, Plast. Rubber. Compos. Process. Appl. 16 (1991) 55.

[24] J. Kolarik, Polym. Compos. 18 (1997) 433.

[25] M. Bornert, C. Stolz, A. Zaoui, J. Mech. Phys. Solids 44 (1996) 307.
[26] E. Hervé, C. Stolz, A. Zaoui, C.R. Acad. Sci. Paris, Série II 313 (1991) 857.

[27] E.H. Kerner, Proc. Phys. Soc. London 69B (1956) 808.

[28] R.M. Christensen, K.H. Lo, J. Mech. Phys. Solids 27 (1979) 315.

[29] E. Hervé, A. Zaoui, Int. J. Engng. Sci. 31 (1993) 1.

[30] M. Bornert, Comput. Mater. Sci. 5 (1996) 17.

[31] Z. Hashin, S. Shtrikman, J. Mech. Phys. Solids 11 (1963) 127.

[32] M. Bornert, E. Hervé, C. Stolz, A. Zaoui, Appl. Mech. Rev. 47 (1) (1994) S66.

[33] F. Aussant, M. Bornert, Internal Report LMS, 2000.

[34] E. Chabert, C. Gauthier, R. Dendievel, L. Chazeau, J.-Y. Cavaillé, in: C.C. Berndt, T.E. Ficher, I. Ovid'ko, G. Skandan, T. Tsakalakos (Eds.), Nanomaterials for Structural Applications, Mat. Res. Soc. Symp. Proc., vol. 740 (2002) 155.

[35] A. Jagota, G.W. Sherer, J. Am. Ceram. Soc. 78 (1995) 521. 